\title{
Infección por Enterobius vermicularis en niños preescolares de Ciudad Bolívar, Venezuela.
}

\section{Artículo Original}

Ixora Requena-Certad ${ }^{1}$, Vishna Lizardi ${ }^{1}$, Luz Mery Mejía ${ }^{1}$, Hector Castillo ${ }^{2}$, Rodolfo Devera ${ }^{1,3}$.

${ }^{1}$ Depto. de Parasitología y Microbiología, Escuela de Medicina, Universidad de Oriente, Núcleo Bolívar, Ciudad Bolívar, Venezuela. ${ }^{2}$ Hospital Universitario "Ruíz y Páez". Ciudad Bolívar, Estado Bolívar, Venezuela. ${ }^{3}$ Depto. de Medicina Tropical, Instituto Oswaldo Cruz, FIOCRUZ. Rio de Janeiro, Brasil.

\section{RESUMEN.}

Introducción. Enterobius vermicularis es un nemátodo con un ciclo biológico particular, donde los huevos se observan en heces sólo en 1 a $5 \%$ de los casos. Es por ello que una técnica diagnóstica especial es requerida: el método de Graham o de la cinta adhesiva.

Objetivo. Determinar la prevalencia de enterobiasis en una muestra de niños en edad preescolar habitantes del área urbana de Ciudad Bolívar, Venezuela.

Material y métodos. Entre Noviembre 1999 y Agosto de 2000 fue realizado un estudio seccional donde fueron evaluados clínica y coproparasitologicamente, mediante el método de Graham, 195 niños entre 2 y 7 años, pertenecientes a cinco institutos públicos de Educación Básica ubicados en el área urbana de Ciudad Bolívar.

Resultados. Se determinó una prevalencia para $E$. vermicularis de $25,6 \%$. No hubo diferencias en cuanto a sexo $\left(\chi^{2}=0,17 ;\right.$ g.l. $=2$ con corrección de Yates) y edad ( $\mathrm{p}>0,05)$ de los niños parasitados. El
$82 \%$ de los infectados estaba sintomático, siendo el prurito anal el hallazgo clínico más frecuente $(68 \%)$, seguido de hiporexia (40\%), intranquilidad (34\%), bruxismo (30\%) y flujo vaginal (20\%).

Conclusiones. Se determinó una elevada prevalencia $(25,6 \%)$ de enterobiasis en niños preescolares. En la mayoría de los casos fue posible determinar un cuadro clínico característico. Debido a las particularidades del ciclo vital de este nemátodo, el examen de rutina de heces no es efectivo por lo tanto ante la sospecha clínica el médico debe solicitar el método de Graham para realizar el diagnóstico.

(Rev Biomed 2002; 13:231-240)

Palabras clave: Parasitosis intestinal, Enterobius vermicularis, preescolares.

\section{SUMMARY.}

Enterobius vermicularis infection in preschool children from Ciudad Bolívar, Venezuela.

Solicitud de sobretiros: Dr. Rodolfo Devera. Departamento de Medicina Tropical, Instituto Oswaldo Cruz. Av. Brasil 4365, Manguinhos, Rio de Janeiro, RJ-Brasil. CEP 21045-900. Telfax: (00-55-21) 280-3740. E-mail: rdevera@dbbm.fiocruz.br Recibido el 28/Mayo/2001. Aceptado para publicación el 8/Enero/2002. 


\section{Requena-Certad, V Lizardi, LM Mejía, H Castillo, R Devera.}

Introduction. The nematode Enterobius vermicularis has a characteristic life cycle in which the eggs are found in the stools in only 1 to $5 \%$ of the cases of infection. For this reason a special technique is required for its diagnosis: the Graham or adhesive tape method.

Objective. To determine the prevalence of enterobiasis in a pre-school children sample from the urban area of Ciudad Bolívar, Venezuela.

Materials and methods. Between November 1999 and August 2000 a cross-sectional survey was performed on 195 children of both sexes aged 2-7, attending 5 public primary schools in the urban area of Ciudad Bolívar. The children were evaluated clinically and coproparasitologically by means of the Graham method.

Results. A prevalence of $25.6 \%$ of E. vermicularis was determined. No differences in relation to age (Yates corrected $\chi^{2}=0.17 ;$ f.g. $\left.=2\right)$ or $\operatorname{sex}(p>0.05)$ were observed between the parasitized children. Eighty two percent of the infected children was symptomatic. Pruritus ani was the most frequent finding $(68 \%)$, followed by hyporexia $(40 \%)$, restlessness (34\%), teeth grinding $(30 \%)$ and vaginal discharge (20\%).

Conclusions. A high prevalence of enterobiasis was observed in pre-school children from Ciudad Bolívar. In most of the cases the characteristic clinical manifestations could be determined. Due to the particularities of this nematode's life cycle, the routine stool exam is not effective for its diagnosis. For this reason, if E. vermicularis infection is suspected at clinical examination, the physician ought to ask for the Graham method for the diagnosis.

(Rev Biomed 2002; 13:231-240)

Key words: Intestinal parasitosis, Enterobius vermicularis, pre-school children, prevalence.

\section{INTRODUCCIÓN.}

Enterobius vermicularis es un nemátodo que causa oxiuriasis o enterobiasis, una de las parasitosis intestinales más frecuentes en todo el mundo (1-7).
E. vermicularis pertenece a la familia Oxiuridae y, junto con la mayoría de los oxiuros de primates, ha sido agrupado recientemente en la nueva subfamilia Enterobiinae (8). El hombre es el único huésped conocido, aunque otras especies de oxiuros parasitan otros vertebrados $(2,4,7,8)$.

La enterobiasis es una de las infecciones parasitarias más antiguas que se conoce, como lo demuestra el hallazgo de huevos del verme en coprolitos de diez mil años de antigüedad $(7,9)$. $E$. vermicularis llegó al continente americano con los inmigrantes que atravesaron el estrecho de Bering $(10,11)$. Hugot y col. (11) sostienen que el nemátodo no fue introducido posteriormente por medio de contactos transpacíficos como afirman otros autores (10).

La parasitosis es más común en grupos, como la familia, escuelas y asilos, siendo más común en niños que en adultos $(2,3)$. Aunque su distribución es mundial (6), en la region tropical la prevalencia es menor, principalmente en comunidades rurales, porque la población usa menos ropa y vive por lo regular en campo abierto, bajo el sol con elevadas temperaturas y baja humedad, donde la supervivencia de los huevos y por tanto la transmisión de la enfermedad es más difícil $(2,3)$.

Este parásito intestinal presenta un ciclo biológico particular donde no se requiere huésped intermedio, ni prolongada incubación exógena para completar el ciclo vital $(3,4)$. Esta peculiaridad determina la posibilidad de ocurrir hasta cuatro mecanismos de transmisión de la parasitosis, lo que explica su elevada prevalencia mundial (2). La manera más frecuente de contaminación es a través de las manos, secundario al rascado de la región perianal. Los huevos también pueden contaminar alimentos y bebidas o ser inhalados o deglutidos directamente del ambiente (2, $3)$. En estudio reciente se ha sugerido que esta transmisión aérea de huevos de E. vermicularis puede tener una gran importancia en el mantenimiento de su ciclo vital (11).

La patología de la enterobiasis depende principalmente de la migración (normal o errática) de los gusanos hembras grávidas y las manifestaciones

\section{Revista Biomédica}


Enterobius vermicularis en preescolares.

clínicas están en relación directa a la carga parasitaria. La mayoría de los individuos que albergan oxiuros están asintomáticos lo que indica que las infecciones leves son las más frecuentes $(2,3,6,12)$. Cuando ocurre el ciclo normal de migración del verme hasta la región perianal ocurre el prurito que es la manifestación más característica de la enfermedad $(2,3,6,7)$. Secundario al rascado de la región anal y perianal pueden ocurrir lesiones mecánicas en esa área e infecciones secundarias, además de las alteraciones del comportamiento frecuentemente observados en niños como consecuencia de las molestias mecánicas que producen los parásitos $(2,3)$. El prurito hace que los niños se despierten por la noche determinado incluso insomnio, además se tornan ansiosos y preocupados por el hecho de ser observados rascándose las regiones anal y genital $(3,13)$. Sumado a estos trastornos en la esfera psicológica puede haber retardo escolar $(13,14)$.

Las infecciones por E. vermicularis también han sido asociadas con una frecuencia incrementada de infecciones en el tracto urinario y genital en niñas (1520). La causa de estas infecciones es la migración de las hembras grávidas para la vagina y uretra. También puede ocurrir migración para otros sitios determinando diversas manifestaciones; sin embargo, las infecciones ectópicas son excepcionales si se considera el gran numero de infectados. Entre las localizaciones ectópicas más frecuentes se reportan la próstata (21), ovarios y trompas de Falopio (21-25), hígado (2628) y el apéndice (6, 29-34). La invasión del apéndice cecal merece especial interés ya que $E$. vermicularis puede ser causante o coadyuvante en casos de apendicitis, bien sea por acción mecánica o por transporte de bacterias y hongos entéricos (29).

Como E. vermicularis sale por el ano a depositar sus huevos, el examen rutinario de heces generalmente detecta al parásito entre 1 a 5\% de los casos. Por ello se requiere de una técnica diagnóstica especial. Desde 1860 hasta 1937 se emplearon instrumentos de todo tipo para extraer del ano, huevos de oxiuros y trasladarlos a un portaobjetos de vidrio para su examen $(2,35)$. En 1937, Hall (36) utilizó una torunda de celofán no adhesiva, denominada comúnmente, torunda N.I.H. (National Institute of Health), y demostró que este método era superior a otros utilizados hasta entonces para obtener huevos de E. vermicularis de la piel perianal. En 1941, Graham (37) introdujo la cinta adhesiva como método diagnóstico y a pesar de haber sufrido algunas modificaciones, actualmente es el método específico usado para diagnosticar $E$. vermicularis $(2,35,37)$. Se recomienda realizar exámenes seriados por lo irregular de las migraciones de las hembras grávidas $(2,4,39)$.

En Venezuela, como en la mayoría de los países de América Latina, la enfermedad es subregistrada, pues los centros y las personas encargadas del diagnóstico no utilizan habitualmente la técnica de Graham. Siendo por tanto, pocos los estudios de prevalencia de parasitosis intestinales que incluyen esta técnica dentro de la metodología. Ciudad Bolívar, como el resto del país, adolece de estudios que determinen la prevalencia real de E. vermicularis, aunque se han realizado investigaciones sobre parasitosis intestinales en la población infantil, pero sin emplear el método de Graham. Apenas 4 trabajos han intentado determinar la prevalencia empleando este método diagnóstico especifico (40-43). Dos fueron realizados en preescolares obteniendo prevalencias de $12 \%$ en 1984 (42) y 8\% en 1991 (43). El tercer estudio fue realizado en guarderías, determinándose un $24 \%$ de infección (41). El último y más reciente trabajo fue realizado por Devera y col. (40) quienes determinaron una prevalencia de $19,1 \%$, entre 282 niños en edad escolar.

Los objetivos del presente estudio fueron: 1) determinar la prevalencia de $E$. vermicularis en una muestra de niños en edad preescolar habitantes de Ciudad Bolívar, utilizando la técnica de Graham;2) conocer la distribución de la parasitosis por edad y sexo de los niños, y 3) estudiar las manifestaciones clínicas para de esta forma contribuir al mejor conocimiento de la epidemiología de la enterobiasis en niños de Venezuela.

\section{MATERIAL Y MÉTODOS.}

Desde noviembre de 1999 hasta agosto de 2000, 


\section{Requena-Certad, V Lizardi, LM Mejía, H Castillo, $R$ Devera.}

fue realizado un estudio seccional utilizando una muestra de niños entre 2 y 7 años, de uno y otro sexo, matriculados en 5 preescolares públicos del área urbana de Ciudad Bolívar en el Estado Bolívar, Venezuela.

1. Selección de la muestra. Para establecer el tamaño de la muestra a utilizar se realizó un estudio piloto en el preescolar Nuestra Señora de Coromoto de Ciudad Bolívar. Para ello se aplicó la técnica de Graham a 48 niños seleccionados aleatoriamente, encontrando huevos de E. vermicularis en 7 (14,6\%) casos. Con base en los resultados del estudio piloto se aplicó la fórmula de Camel y Schuartz (44) para estimar el número de niños necesarios para determinar la prevalencia de E. vermicularis en la población preescolar:

$$
\mathrm{n}=\frac{\left(\mathrm{Z}^{2}\right)(\mathrm{p})(\mathrm{q})}{\mathrm{i}^{2}}
$$

Donde:

n: número de sujetos necesarios para determinar la prevalencia de $E$. vermicularis

Z: 1,96 (valor tomado de la distribución normal estandarizada).

p: porcentaje de preescolares positivos en el estudio piloto.

q: porcentaje de preescolares negativos en el estudio piloto.

i: error tolerado por el investigador el cual fue de $5 \%$.

Sustituyendo los valores en la fórmula se determinó en 191 el número de preescolares necesarios.

En Ciudad Bolívar, como en toda Venezuela, la educación preescolar esta dividida en tres niveles: I, II y III, correspondiendo respectivamente a niños de 3,4 y 5 años de edad. Sin embargo, no es infrecuente encontrar niños con 2 años en el primer nivel así como niños con 6 y 7 anos en el último nivel.

Para seleccionar los 191 niños, el área urbana de Ciudad Bolívar fue dividida en cuatro regiones seleccionándose una institución en cada una de ellas. El preescolar que había sido escogido previamente para hacer el estudio piloto también fue incluido. Para prevenir posibles pérdidas (no-asistencia o nocumplimiento de los criterios de inclusión) se sobrestimó en $15 \%$ el número de los niños a ser evaluados. Para la selección, en cada institución fueron escogidos aleatoriamente 45 niños ( 15 de cada nivel).

\section{Aplicación de la técnica de Graham:}

a. Condiciones previas. En cada una de las 5 instituciones se realizaron visitas previas donde se informó sobre la importancia del estudio a docentes, padres y representantes. Se entregó una citación a cada alumno, indicando las condiciones para realizar el estudio. Se obtuvo el consentimiento por escrito del representante del alumno. El niño no debía haberse bañado, defecado o realizado aseo perianal el día indicado para realizar el procedimiento. Además se investigó el uso de drogas antiparasitarias, excluyéndose a todos aquellos niños que las recibieron un mes antes o durante el estudio.

b. Realización de la técnica. La toma de las muestras se realizó en la presencia de los padres de 7 a 8:30 de la mañana. Se practicó un interrogatorio a cada representante, obteniendo datos de identificación y manifestaciones clínicas actuales del preescolar. Además, se realizó un examen físico a cada niño. Se recolectó una muestra por frotamiento de la región perianal con cinta adhesiva transparente, la cual se colocó sobre un portaobjetos previamente identificado. La búsqueda de los huevos y/o adultos de E. vermicularis se realizó mediante examen microscópico de los portaobjetos con objetivo de $10 \mathrm{X}$.

Por razones éticas y a petición de los representantes también fueron realizados exámenes en niños que no fueron seleccionados para el estudio.

\section{Análisis estadístico.}

La prevalencia fue calculada según Morales y Pino (44). En el análisis estadístico de los resultados fueron utilizadas frecuencias relativas $(\%)$. Para estudiar la relación entre infección por $E$. vermicularis y la edad de los preescolares se aplicó la prueba Chi-cuadrada $\left(\chi^{2}\right)(44)$.

\section{Revista Biomédica}


Enterobius vermicularis en preescolares.

Cuadro 1.

Preescolares parasitados con E. vermicularis, según edad y género. Ciudad Bolívar, Venezuela. 1999-2000.

\begin{tabular}{|c|c|c|c|c|c|c|c|c|c|}
\hline \multirow{4}{*}{$\begin{array}{l}\text { Grupo } \\
\text { etáreo } \\
\text { (años) }\end{array}$} & \multicolumn{6}{|c|}{ Género } & \multirow{2}{*}{\multicolumn{3}{|c|}{ Total }} \\
\hline & Feme & & & \multicolumn{3}{|c|}{ Masculino } & & & \\
\hline & \multirow{2}{*}{$\begin{array}{l}\text { Total } \\
\text { evaluados }\end{array}$} & \multicolumn{2}{|c|}{ Parasitados } & Total & Par & dos & Evaluados & Par & tados \\
\hline & & No. & $\%$ & evaluados & No. & $\%$ & & No. & $\%$ \\
\hline $2-3$ & 9 & 2 & 22,2 & 13 & 3 & 23,1 & 22 & 5 & 22,7 \\
\hline $4-5$ & 90 & 18 & 20,0 & 66 & 23 & 34,8 & 156 & 41 & 26,3 \\
\hline $6-7$ & 9 & 3 & 33,3 & 8 & 1 & 12,5 & 17 & 4 & 23,5 \\
\hline Total & 108 & 23 & 21,3 & 87 & 27 & 31,0 & 195 & 50 & 25,6 \\
\hline
\end{tabular}

$\chi^{2}=0,17 \quad$ g.l. $=2 \quad \mathrm{p}>0,05$

RESULTADOS.

De los niños convocados, asistieron 198 siendo excluidos tres por no cumplir las condiciones establecidas.

El 55,4\% (108/195) era del género femenino y el 44,6\% (87/195), del masculino. Un total de 50 escolares estaba parasitado con E. vermicularis, lo que representa una prevalencia de $25,6 \%$. El género masculino fue el más afectado, con 54\% (27/87), pero esa diferencia no fue estadísticamente significativa $(\mathrm{p}$ $>0,05)$. En el grupo etáreo de 4 a 5 años se observó el mayor número de casos $(41 / 50)$, pero esa diferencia tampoco fue significativa $\left(\chi^{2}=0,17\right.$ g. $=2$, con corrección de Yates) pues en este grupo estaba el mayor numero de evaluados (cuadro 1).

Además de E. vermicularis se diagnosticó Ascaris lumbricoides en 14 casos (7,2\%) y Trichuris trichiura en $5(2,6 \%)$.

El 67,7\% (132/195) de los evaluados presentaba sintomatología sugestiva de alguna parasitosis intestinal. Entre los sintomáticos, 41 $(31,1 \%)$ estaban infectados con E. vermicularis. La relación infección por $E$. vermicularis y presencia

Cuadro 2.

Preescolares parasitados o no con $E$. vermicularis, según sintomatología. Ciudad Bolívar, Venezuela. 1990 - 2000.

\begin{tabular}{llllllll}
\hline \multirow{2}{*}{ Preescolares } & \multicolumn{3}{c}{ E. vermicularis } & \multicolumn{4}{c}{ Total } \\
& No. & Si & No. & $\%$ & No. & $\%$ \\
\hline Sintomáticos & 41 & 31,1 & 91 & 68,2 & 132 & 67,7 \\
Asintomáticos & 9 & 14,3 & 54 & 85,7 & 63 & 32,3 \\
Total & 50 & 25,6 & 145 & 74,4 & 195 & 100,0 \\
\hline$\chi^{2}=6,29 ;$ & g.l. $=1$ & $\mathrm{p}<0,05$ & & &
\end{tabular}

de síntomas en los niños parasitados fue significante $\left(\chi^{2}=6,29 ;\right.$ g.l. = 1) (cuadro 2). El prurito anal fue la manifestación clínica más frecuente $(68 \%)$, seguido de hiporexia (40\%), intranquilidad (34\%), bruxismo (30\%) y flujo vaginal (20\%). Otras manifestaciones infrecuentes, agrupadas como otras, fueron: cefalea, diarrea, astenia y pérdida de peso, encontrándose un caso con cada una de ellas (cuadro 3).

El prurito anal resulto más frecuente en el género masculino con $36 \%$ (18/50), contra $28 \%$ (14/50) en el género femenino; sin embargo, esta asociación no fue significativa $\left(\chi^{2}=0,18 ;\right.$ g.l. $\left.=1\right)$. El 74\% (37/ 50) de los parasitados tenía hermanos en el hogar con prurito anal.

El 70\% (35/50) de los parasitados con $E$. vermicularis, había recibido tratamiento antihelmíntico específico previo. En el grupo que resultó negativo para E. vermicularis, 51\% (74/145), había usado antihelmintico (cuadro 4).

Cuadro 3.

Manifestaciones clínicas en 50 preescolares parasitados con E. vermicularis. Ciudad Bolívar, Venezuela, 1999 - 2000.

\begin{tabular}{lcccc}
\hline $\begin{array}{l}\text { Manifestaciones } \\
\text { clínicas }\end{array}$ & \multicolumn{2}{c}{ Género } & \multicolumn{2}{c}{ Total } \\
& Masculino & Femenino & No. & $\%$ \\
\hline Prurito anal & 16 & 18 & 34 & 68 \\
Hiporexia & 13 & 7 & 20 & 40 \\
Intranquilidad & 10 & 7 & 17 & 34 \\
Bruxismo & 7 & 8 & 15 & 30 \\
Flujo vaginal & - & 10 & 10 & 20 \\
Insomnio & 3 & 5 & 7 & 14 \\
Otras* & 2 & 2 & 4 & 8 \\
& & & & \\
\hline
\end{tabular}

*Cefalea, diarrea, astenia y pérdida de peso.

Vol. 13/No.4/Octubre-Diciembre, 2002 


\section{Requena-Certad, V Lizardi, LM Mejía, H Castillo, R Devera.}

\section{Cuadro 4}

Preescolares parasitados o no con $E$. vermicularis, según tratamiento antihelmíntico específico previo. Ciudad Bolívar, Venezuela. 1999 - 2000.

\begin{tabular}{lcccccc}
\hline & \multicolumn{3}{c}{ Tratamiento previo* } & & \\
Preescolares & \multicolumn{2}{c}{ Si } & \multicolumn{2}{c}{ No } & \multicolumn{2}{c}{ Total } \\
& No. & $\%$ & No. & $\%$ & No. & $\%$ \\
\hline No Parasitados & 74 & 51 & 71 & 49 & 145 & 74,4 \\
Parasitados & 35 & 70 & 15 & 30 & 50 & 25,6 \\
Total & 109 & 55,9 & 86 & 44,1 & 195 & 100,0 \\
\hline$\chi^{2}=5,42 ;$ g.l. = 1 & $\mathrm{p}<0,05$ & & & \\
$*$ Pamoato de Pirantel, Mebendazol o Albendazol &
\end{tabular}

\section{DISCUSIÓN.}

Se estima que entre 20 y $30 \%$ de los niños en el ámbito mundial presentan infección por $E$. vermicularis $(1,13,35)$. En Estados Unidos es el parásito intestinal más frecuentemente diagnosticado desde hace varios años, siendo la prevalencia más reciente de $11,4 \%$ (45).

Desde noviembre de 1999 hasta julio de 2000 se evaluaron 195 preescolares de Ciudad Bolívar, pertenecientes a cinco institutos de Educación Básica ubicados en el área urbana. De ellos, 108 eran hembras y 87 varones. Se determinó una prevalencia de 25,6\% de enterobiasis, lo que representa 50 niños parasitados demostrado por el método de Graham. E. vermicularis es más prevalente en instituciones con régimen de internado, como las escuelas $(1,13$, 35); por lo tanto, se esperaba una prevalencia elevada en el grupo estudiado. Tal vez el empleo de apenas una única toma de muestra para examen contribuyó a subestimar el diagnóstico. Se había planificado tomar 3 muestras seriadas a cada niño sin embargo, la poca colaboración de los padres y representantes obligó a realizar un examen único por preescolar. Sin embargo, los resultados son similares a los reportados previamente por otros autores $(40,46)$.

Además de E. vermicularis fueron encontrados A. lumbricoides y T. trichiura. El hallazgo de huevos de otros helmintos en la cinta adhesiva es frecuente (37, 38, 40, 43), sugiriendo un intenso poliparasitismo de la población evaluada.

En niños en edad preescolar las prevalencias señaladas de enterobiasis son muy variables de un país a otro. En 1983, Wagner y Eby (46) determinaron una prevalencia de $25,9 \%$ en 5 escuelas de California en los Estados Unidos. Chan (47) reportó 31,5\% en la provincia portuguesa de Macao en la costa sur de China y en Iraq se señala una baja tasa de infección de $9 \%$ (48). Datos más recientes, muestran prevalencias de $7,8 \%$ en Corea (12) y de $14 \%$ en Taiwan (49). Estos datos son pocos comparables debido al diferente tamaño de las muestras utilizadas.

En la mayoría de los países latinoamericanos, las tasas de prevalencia también son variables, aunque generalmente son elevadas. En Guatemala oscila entre un 50 y $70 \%$ (13); en Perú, Gilman y col. (50) estudiando una comunidad de Lima señalaron $42 \%$. En Chile reportes recientes señalan 20,9\% (5) y en Cuba 28\% (51). Algunas de estas altas tasas de prevalencias contrastan con la obtenida en nuestro estudio.

En Venezuela, la prevalencia exacta de enterobiasis no es conocida. Romero de Olaria (38), estudiando la población infantil hospitalizada en un gran hospital de Maracaibo, en el occidente del país, señaló un 7,3\%. Greatty y col. (52) en el Estado Anzoátegui, determinaron una prevalencia en preescolares de $49,1 \%$ que es mayor a la encontrada en el presente estudio.

En la población preescolar de Ciudad Bolívar, los datos conocidos indican $12 \%$ en 1984 (42) y $8 \%$ en 1991 (43). Siendo ambos estudios realizados en grupos seleccionados y con un número reducido de individuos. Esas prevalencias son inferiores a las encontradas en el presente estudio.

La parasitosis resultó más frecuente en varones, pero esta diferencia no fue significativa $(p>0,05)$, coincidiendo con otros estudios $(5,38-40,42,46-$ $48,52)$. Tal vez la adquisición de normas de higiene más precozmente en las niñas explica esta diferencia. El grupo etáreo más afectado fue el de 4 y 5 años, siendo esta diferencia estadísticamente no significativa

\section{Revista Biomédica}


ya que el $80,4 \%$ de los niños evaluados pertenecía a este grupo etáreo. Resultados similares fueron reportados previamente $(42,46)$. Otros autores mostraron que la parasitosis es significativamente más prevalente en niños de más edad $(39,47,48,50$, 52).

Con relación a las manifestaciones clínicas, $67,7 \%$ de los preescolares evaluados presentaban manifestaciones clínicas compatibles con enterobiasis, pero sólo 41 casos (31\%) fueron confirmados mediante el hallazgo de los huevos característicos en la cinta adhesiva. Russell (7) señala que es necesario el diagnóstico confirmatorio de la enfermedad pues, no hay diferencia en cuanto a presencia de sintomatología en niños infectados y no infectados. En el presente estudio se demostró que los niños infectados con $E$. vermicularis estaban más sintomáticos que el grupo no parasitado, comprobándose que generalmente hay una correlación entre sintomatología y enterobiasis $(40,53)$. Cuando la parasitosis es intensa y el diagnóstico es bien orientado suelen encontrarse los síntomas. De allí que más del $80 \%$ de los niños presentaban alguna sintomatología. El síntoma referido con mayor frecuencia fue el prurito anal. Estudios previos han mostrado que éste es característico de la infección por E. vermicularis $(2,3,6,7,21,40,50,52)$ y excepcionalmente otros síntomas predominan en los niños infectados (53). Al igual que otros estudios, los síntomas nerviosos ocuparon el segundo lugar de presentación $(40,52)$.

El bruxismo es un dato inespecífico de enterobiasis, pero es reportado frecuentemente $(1,40$, 52 ), como en nuestro estudio que tuvo una frecuencia de $30 \%$.

En diez niñas (20\%) se observó flujo vaginal. La vulvovaginitis infantil y por lo tanto el flujo, puede ser causado por agentes infecciosos y no infecciosos, ninguno de los cuales fueron investigados por no ser parte de los objetivos del estudio. Sin embargo, otros autores han señalado la importancia de $E$. vermicularis como agente de vulvitis, ya sea por su propia acción y/o como transportador de bacterias entéricas a la zona genital $(1,7,13,16,17,20,35$,
40). En Venezuela, Brito y col. (16), demostraron que $34,3 \%$ de las escolares evaluadas tenía vulvitis asociada con E. vermicularis y Devera y col. (40) reportaron una frecuencia de $26,1 \%$ entre 23 niñas en edad escolar. En las Islas Canarias, E. vermicularis supera a las bacterias como causa de vulvovaginitis en niñas prepúberes con un 12,6\% (20).

Otro aspecto a considerar en la epidemiología de la enterobiasis es su facilidad de transmisión. Al investigar sobre la presencia, en el mismo grupo familiar, de hermanos con prurito anal, se obtuvo que $28 \%$ de los parasitados referían este antecedente, coincidiendo con lo señalado por otros autores $(2,7$, $40,50)$ y confirmando el carácter familiar del prurito $(1,2,7,35)$.

Con relación al uso de terapia antihelmíntica previa, por parte de los preescolares, cabría esperar que entre los parasitados este antecedente fuera menos frecuente y visceversa, entre los no parasitados (40). Sin embargo, lo encontrado fue contradictorio. De los 195 evaluados, 55,9\% había recibido drogas antihelmínticas tres meses antes del estudio y dentro de los parasitados, el 70\% informó haber recibido tratamiento antihelmíntico previo. Entre los no parasitados, $51 \%$ habían usado tratamiento previo al estudio. Esto permite realizar dos consideraciones: primero, si el tratamiento previo fue efectivo pueden haber sucedido reinfecciones en esos niños, lo cual frecuentemente ocurre (51); y segundo, tal vez la terapia no haya sido efectiva por diversas causas (uso de dosis o esquemas de tratamiento inadecuados, automedicacion, etc). Cualquiera que sea la causa de estos hallazgos, ambas sugestiones podrían explicar la elevada tasa de enterobiasis en el grupo que recibió tratamiento antihelmíntico en los tres meses que precedieron al estudio.

En conclusión se determinó una relativa alta prevalencia $(25,6 \%)$ de E. vermicularis en preescolares de Ciudad Bolívar. El médico con base en los datos clínicos y antecedentes sugestivos de enterobiasis debe solicitar el examen de la cinta adhesiva (método de Graham) que es específico para hacer el diagnóstico de esta helmintiasis. Se debe considerar que, si bien los casos graves son raros, las 


\section{Requena-Certad, V Lizardi, LM Mejía, H Castillo, R Devera.}

alteraciones en la esfera psicológica sí suelen serlo, conduciendo a una situación de minusvalía en el niño, con la consecuente afectación de su salud y de los procesos de enseñanza-aprendizaje.

\section{REFERENCIAS.}

1.- Atías A. Enterobiasis u Oxiurasis. En: Atías A, Negme A, editores. Parasitología Clínica. 3ra. ed. Santiago: Publicaciones Técnicas Mediterráneo; 1993. p. 184-89.

2.- Beaver JJ, Jung RC, Cupp EV. Clinical Parasitology. 9th. ed. Philadelphia: Lea and Febiger; 1984.

3.- Botero D, Restrepo M. Parasitosis humanas. 3ra. ed. Medellin: Corporación para Investigaciones Biológicas; 1998.

4.- Brown WH. Parasitología Clínica. 4ta. ed. Mexico: Nueva Editorial Interamericana SA; 1975

5.- Mercado R, García M. Algunos aspectos epidemiológicos de las infecciones por Enterobius vermicularis en pacientes atendidos en consultorios de atención primaria del sector norte de Santiago, 1995. Bol Chil Parasitol 1996; 51: 91-4.

6.- Organización Mundial de la Salud. Infecciones Intestinales por protozoos y helmintos. Informe de un grupo científico de la O.M.S. Serie 660. España: Editorial Gráficas Reunidas. 1981.p. 155.

7.- Russell LJ. Enfermedades Parasitarias. Clin Aten Prim 1991; 1:14-25.

8.- Hugot JP, Gardner SL, Morand S. The Enterobiinae fam. nov. (Nematoda, Oxyurida), parasites of primates and rodents. Inter J Parasitol 1996; 26: 147-59.

9.- Fry GF, Moore JG. Enterobius vermicularis: 10,000 year old human infection. Science 1969; 166: 1620.

10.- Araujo JG, Ferreira LF, Confalorieri EL, Nuñez L, Ribeiro Filho BM. The Finding of Enterobius vermicularis eggs in pre-columbian coprolites. Mem Inst Oswaldo Cruz 1985; 80:141-3.

11.- Hugot JP, Reinhard KJ, Gardner SL, Morand S. Human Enterobiasis in evolution: origin, specifity and transmission. Parasite 1999; 6: 201-8.

12.- Yoon HJ, Choi YJ, Lee SU, Park HY, Huh S, Yang YS. Enterobius vermicularis egg positive rate of pre-school children in Chunchon, Korea (1999). Korean J Parasitol 2000;
38: $279-81$.

13.- Aguilar FJ. Parasitología Clínica. Guatemala: Litografía Delgado, SA;1991.

14.- Behader SM, Ali GS, Shaalan AH, Khalil HM, Khalil NM. Effects of Enterobius vermicularis infection on intelligence quotient (I.Q) and anthropometric measurements of Egyptian rural children. J Egypt Soc Parasitol 1995; 25:18394.

15.- Al-Rufaie HK, Rix GH, Pérez-Clemente MP, Al-Shawaf T. Pinworms and potmenopausal bleeding. J Clin Pathol 1998; 51:401-2.

16.- Brito A, Rodríguez MC, Berdeal E, Verdey M, López Y, Farías Z. et al. Vulvovaginitis Infantil: manifestaciones, etiología y eficacia terapéutica del sulfametoxazole/ trimetropin y la yodo povidona. Bol Soc Venezolana Microbiol 1994; 4: 6-14.

17.- Gokalp A, Gultekin EY, Kirisci MF, Ozdamar S. Relation entre Enterobius vermicularis infestation and dysuria, nocturia, enuresis nocturna and bacteriuria in primary school girls. Indian Pediatr 1991; 28:948-50.

18.- Kropp KA, Cichocki GA, Bansal NK. Enterobius vermicularis (pinworms), introital bacteriology and recurrent urinary infections in children. J Urol 1978; 120: 480.

19.- Ok UZ, Ertan P, Limoncou E, Ece A, Ozbakkaloglu B. Relationship between pinworm and urinary tract intections in young girls. APMIS 1999; 107: 474-6.

20.- Pena MJ, Campos-Herrero MI, Ruíz MC, Rodriguez H, Lafarch B. Microbiological study of vulvo vaginitis in premeraharcheal girls. Enferm Infecc Microbiol 1996; 14: 3113.

21.- Symmers W. Pathology of oxyuriasis. Arch Pathol 1950; 50: 475-516.

22.- Beckman E, Holland JB. Ovarian enterobiasis- a proposed pathogenesis. Am J Trop Med Hyg 1981; 30: 74-6.

23.- Croce EJ, MacGillivray WF, Murphy CJ. Salpingitis due to Enterobius vermicularis. N Eng J Med 1956; 254: 67-9.

24.- Saffos RO, Rhatigan RM. Unilateral salpingitis due to Enterobius vermicularis. Am J Clin Pathol 1977; 67: 296-99.

25.- Vasquez-Piloto A, Cruz-Robaina JC, Nuñez-Fernández F, Sánchez-Díaz JM. Absceso tubo-ovárico bilateral debido 


\section{Enterobius vermicularis en preescolares.}

a granulomas por Enterobius vermicularis. Presentacion de un caso. Rev Cubana Med Trop 1994; 46: 65-7.

26.- Daley JJ, Baker GF. Pinworm granuloma of the liver. Am J Trop Med Hyg 1984; 33:62-4.

27.- Litle MD, Cuello JC, D’Alessandro A. Granuloma of the liver due to Enterobius vermicularis. Am J Trop Med Hyg 1973; 22:567-9.

28.- Mondou EN, Gnepp DR. Hepatic granuloma resulting from Enterobius vermicularis. Am J Clin Pathol 1989; 91: $97-$ 100.

29.- Dorfman S, Talbot IC, Torres R, Cardozo J, Sánchez M. Parasitic infestation in acute appendicitis. Ann Trop Med Parasitol 1995; 89: 99-101.

30.- Ajao OG, Jastaniah S, Malatani TS, Morad N, El Tayed EN, Saif SA, et al. Enterobius vermicularis (pin worm) causing syptoms of appendicitis. Trop Doc 1997; 27:182-3.

31.- Dalhstrom JE, Macarthur EB. Enterobius vermicularis: a possible cause of symptoms resembling appendicitis. Aust NZ J Surg 1994; 64: 992-4.

32.- Rojas C. Infección parasitaria apendicular por Enterobius vermicularis. Bol Chil Parasitol 1959; 14: 26-7.

33.- Sinniah B, Leopairut J, Neafie RC, Connor DH, Voge M. Enterobiasis: a histopathological study of 259 patientes. Ann Trop Med Parasitol 1991; 85: 625-35.

34.- Sterba J, Vlcek M. Appendiceal enterobiais- its incidence and relationsships to appendicitis. Folia Parasitol 1984; 31 : 311-8.

35.- Mathies J. Nematodiasis Intestinal. In: Hoeprich E. editor. Tratado de Enfermedades Infecciones. 2da ed. Mexico Salvat Editores; 1982. p. 590-643.

36.- Hall MC. Studies on oxyuriasis. I. Types of anal swabs and scrapers, with a description of an improved type of swab. Am J Trop Med 1937; 7: 445-53,

37.- Graham CF. A Device for the Diagnosis of Enterobius infection. Am J Trop Med 1941; 21: 159-61.

38.-Romero de Olaria T. Estudios sobre la incidencia de Enterobiasis en niños del Hospital Universitario de Maracaibo, utilizando el Método de Graham modificado por Jacobs. Kasmera 1976; 7: 95-115.
39.- Norhayati M, Hayati MI, Oothuman P, Azizi O, Fatmah MS, Ismail G, et al. Enterobius vermicularis infection among children aged 1-8 years in a rural área in Malaysia. South Asian J Trop Med Pub Health 1994; 25: 494-8.

40.- Devera R, Pérez C, Ramos Y. Enterobiasis en escolares de Ciudad Bolívar, Estado Bolívar. Venezuela. Bol Chil Parasitol 1998; 53: 14-8.

41.- Guevara R. Evaluación de la población infantil en los hogares de cuidado diario de Ciudad Bolívar. Diagnostico de salud. En: II Congreso Científico de la Universidad de Oriente 1992; Nov 5-9; Cumaná, Venezuela. vol. 1:348.

42.- Hernández I, Guevara R. Incidencia de Enterobius vermicularis en la población preescolar de Ciudad Bolívar. Estado Bolívar. Act Cient Vzlana 1984; 35(suppl 1): 380.

43.- Segovia I. Parasitismo Intestinal y su repercusión sobre el déficit pondoestatural en Preescolares del plantel J.M. Siso Martínez residentes en la Urbanización Aceiticos I. La Sabanita. Ciudad Bolívar. [Tesis]. Ciudad Bolívar: Universidad de Oriente; 1991.

44.- Morales G, Pino LA. Parasitología cuantitativa. Caracas: Fondo Editorial Acta Cientifica Venezolana; 1987. p 132.

45.- Kappus KD, Lundgren RG, Juranek, DD, Roberts JM, Spencer HC. Intestinal parsitism in the United States: update on a continuing problem. Am J Trop Med Hyg 1994; 50: 705-13.

46.- Wagner ED, Eby W. Pinworm prevalence in California elementary school children, and diagnostic methods. Am J Trop Med Hyg 1983; 32: 998-1001.

47.- Chan CT. Enterobiasis among school children in Macao. Southeast Asian J Trop Med Pub Health 1985; 16: 547-53.

48.- Mahdi NK, Al-Khafaji A. Prevalence and seasonal variation of enterobiasis in children of Iraq. Southeast Asian J Trop Med Pub Health 1990; 21:135-8.

49.- Fan PC. Review of enterobiasis in Taiwan and offshore islands. J Microbiol Immunol Infect 1998; 31: 203-10.

50.- Gilman RH, Marquis GS, Miranda E. Prevalence and symptoms of Enterobius vermicularis infections in a Peruvian shanty town. Trans R Soc Med Hyg 1991; 85: 7614.

51.- Nuñez FA, Hernández M, Finlay CM. A longuitudinal study of enterobiasis in three day care centers of Havana

Vol.13/No.4/Octubre-Diciembre, 2002 


\section{Requena-Certad, V Lizardi, LM Mejía, H Castillo, R Devera.}

City. Rev Inst Med Trop São Paulo 1996; 38: 129-32.

52.- Greatty O, González C, Sánchez M, Morocoima A. Incidencia de Enterobiasis en niños de una población del Estado Anzoátegui: obtenido a través del Método de Graham. Act Cient Vzlana 1994; 43(suppl 1): 263.

53.- López C, Villanueva C. Enterobiasis en la población estudiantil del Distrito de Subtan-Jalla I.C.A. En: XI Congreso Latinoamericano de Parasitología 1993; Oct 11-17; Lima, Perú. p.100. 